\title{
OFRENDAS, ASENTAMIENTOS Y HUMEDALES: SISTEMAS DE CONTROL TERRITORIAL EN EL OCCIDENTE DE LA PENÍNSULA IBÉRICA
}

\author{
OFFERINGS, SETTLEMENTS AND WETLANDS: SYSTEMS OF \\ TERRITORIAL CONTROL IN WESTERN IBERIA
}

\author{
por
}

BEATRIZ DÍAZ SANTANA

RESUMEN La transición del Bronce Final al Primer Hierro en la fachada atlántica se corresponde en el registro arqueológico con la desaparición paulatina de depósitos o representaciones de objetos de prestigio en el paisaje cultural. Su deposición es el referente visible en el paisaje de grupos con una ganadería transterminante y no sedentarizados. En el Bronce Final surge una aristocracia cuyos elementos de prestigio constituyen los depósitos hallados, que podrían tener carácter funerario. Los valles de los ríos y sus humedales son un elemento clave a la hora de entender la dispersión y significado de asentamientos y hallazgos.

ABSTRACT Archaeological remains in Later Bronze Age - Iron Age transition reveal the ending of luxury objects deposits in the cultural landscape. These depositions are contextualized in landscape domain as references of groups with "transterminance" livestock and without sedentarism. These luxury items belong to an aristocracy which emerged from the Later Bronze Age, and may have had funerary meanings. River valleys and wetlands are valuable references when understanding settlements and deposits dispersion.

\section{INTRODUCCIÓN. EL PANORAMA EUROPEO}

El Bronce Final de la Europa atlántica ve aparecer en el registro arqueológico de forma recurrente depósitos de armas y objetos de prestigio vinculados a las aguas, ya sea por haber sido arrojados a estas, o por hallarse en zonas húmedas (fens, turberas...) (Bradley 1990; Ruiz-Gálvez 1995b). Existen diferencias contextuales e intencionales en los depósitos de la Europa Nórdica respecto a los de la Atlántica, en la que situamos el Occidente Peninsular (Bradley 1990; Ruiz-Gálvez 1995c). Al mismo tiempo, los datos funerarios parecen 
desaparecer por completo, sobre todo en el caso de Galicia, a mediados del II milenio a.C. (Fábregas \& Bradley,1995; Fábregas y Ruiz-Gálvez 1997).

La emergencia de los primeros asentamientos estables, que sucede de forma anacrónica en la fachada atlántica, se corresponde en el registro arqueológico con la paulatina desaparición de estos depósitos, o un cambio en su significado y contexto (Bradley 1990).

Estos datos parecen indicar variaciones en la concepción del espacio cultural pasando de un control del paisaje mediante la deposición de armas, ya sea en las aguas o en puntos nodales de las vías de comunicación, por parte de sociedades con un carácter móvil, a la reivindicación del territorio materializada en el asentamiento (Bradley 1990; Ruiz-Gálvez 198219901991 1995b; Galán 1993; Parcero 1995).

\section{RITUALES DE MUERTE Y PODER}

La tradición cultural de hacer ofrendas a las aguas, sobre todo a los ríos, es una constante en la Europa Atlántica desde el Neolítico, reemplazándose posteriormente los objetos en piedra o madera por el metal durante la Edad del Bronce. Los objetos que se depositan también varían, tanto porque varía su simbología como los contextos sociales y económicos en que se realizan las deposiciones (Bradley 1990).
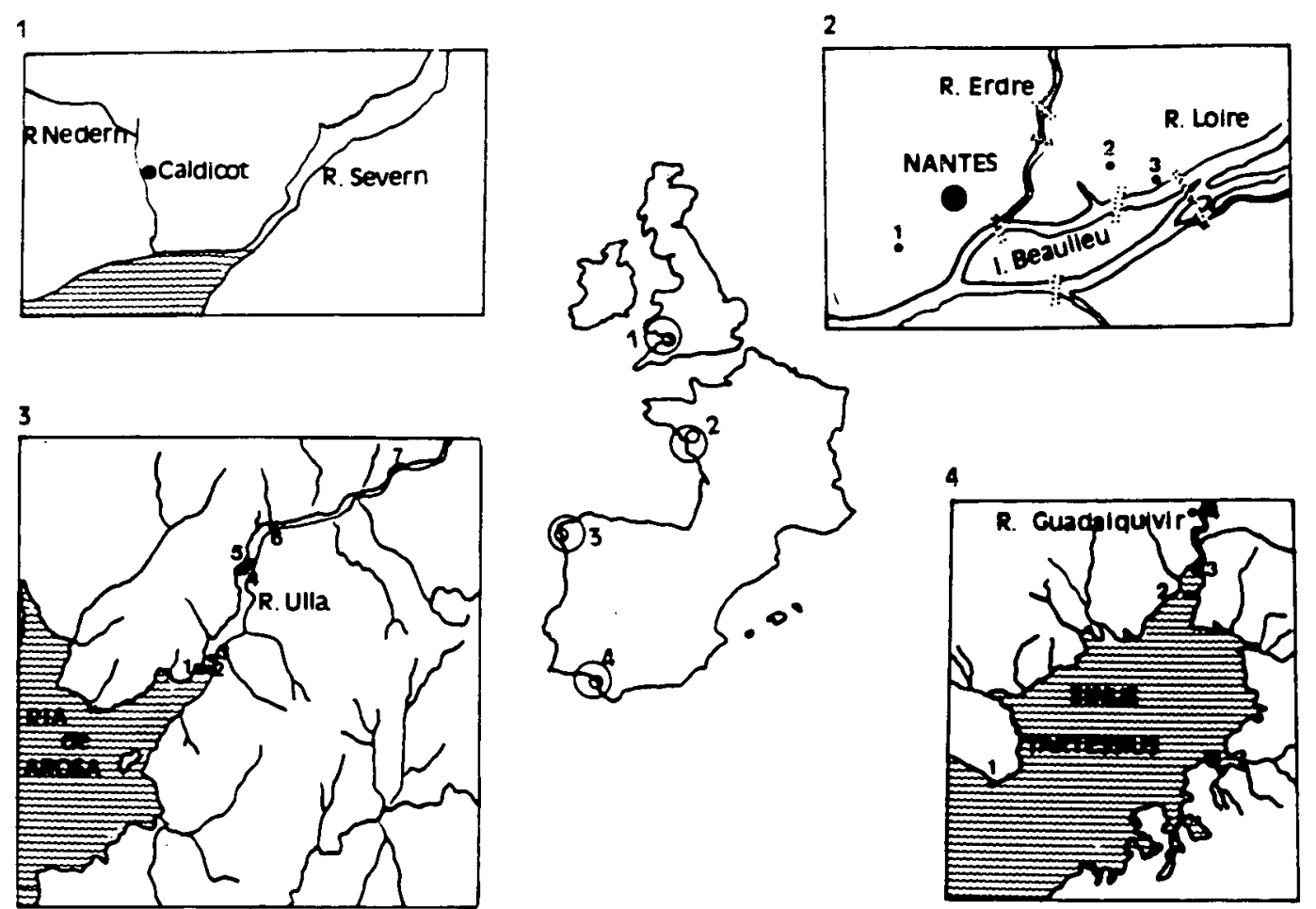

Figura 1: Sitios de la Edad del Bronce en la desembocadura de los ríos (según Ruiz-Gálvez 1995b): - 1. Caldicot, según Parry \& MacGrail 1991. - 2. Nantes según Briard et al. 1966 - 3. Hallazgos en la desembocadura del Ulla. 4. Desembocadura del río Guadalquivir. 
En la Europa Nórdica, estos depósitos se realizan con objetos derivados de contextos rituales, como trompetas (lures), mientras que en la costa atlántica, serán las armas el objeto más depositado (hachas, espadas , escudos ...) (Bradley 1990; Fábregas \&Bradley 1995; Ruiz-Gálvez,1982,1995a 1995b y 1995c).

La ausencia aparente durante buena parte del Bronce Final de restos vinculados a la esfera funeraria encuentra según algunos autores una explicación en la desviación de los objetos que acompañaban al difunto, de los ajuares de los túmulos y cistas, hacia las aguas (Bradley 1990; Fábregas\&Bradley 1995). Se han hallado numerosos restos humanos en los ríos, en ocasiones acompañando a las armas depositadas. Es el caso de los fragmentos de cráneos del Támesis (Bradley\&Gordon 1988) o de Fengate Power Station (Pryor 1992). Los restos humanos, tras un descarnamiento inicial, serían arrojados parcialmente a las aguas, predominando ciertas partes anatómicas, sobre todo los cráneos (Bradley\&Gordon 1988; Fábregas y Bradley 1995).

No todos los individuos serían tratados por igual. Los objetos depositados, sobre todo armas y adornos, todos ellos objetos de prestigio, indican una jerarquización social, encabezada por las élites de carácter guerrero. M.A. Aldhouse Green (1993), vincula estos depósitos a una concepción religiosa propia del mundo celta, lo mismo que M. Almagro Gorbea para la Península Ibérica (1986 1993 y 1993a).

En la Península Ibérica no han sido encontrados restos humanos asociados a depósitos, ni en la Ría de Huelva (Ruiz-Gálvez 1995b), ni con las espadas de los ríos de Galicia (Ruiz-Gálvez 197819821995 b y e.p.-a). Sólo en la Meseta Norte, en los yacimientos tipo Cogotas I, la triple inhumación de San Román de Hornija, el enterramiento no bien documentado de Renedo de Esgueva o la sepultura palentina de Carralasvegas (Santillana de Campos), han aparecido restos humanos, que pueden ser considerados una práctica funeraria de desarrollo autóctono (Delibes et al. 1995: 49-59), pero no es seguro que se trate de enterramientos propiamente dichos, destacando su carácter excepcional y su ubicación en silos de almacenaje o basureros, con escasas evidencias de ajuar y probablemente no ajustados a un ritual propiamente dicho (Romero y Jimeno 1993; Delibes et al. 1995: 49-59; Almagro 1986). La presencia en San Román de Hornija de una fíbula de codo tipo Ría de Huelva, y de un torques tipo Tara en Castrojeriz (Burgos), sitúa la Cultura de Cogotas I dentro de amplias redes de intercambio durante el Bronce Final I-II (Delibes et al. 1995: 59).

La Ría de Huelva, según M. Ruiz-Gálvez (1995a, b y c), corresponde a un ritual similar a los documentados en el resto de la Europa Atlántica del Bronce Final II-III. Datado en el 950 A.C. (Ruiz-Gálvez 1995d), se compone mayoritariamente de objetos de prestigio (casco de procedencia oriental, fíbulas, armas), que serían depositados en ceremonias rituales con el doble fin de amortizar el bronce para revalorizarlo, y para mantener el estatus del oferente. Restos de madera hallados en el depósito, y que no se han conservado, plantean la cuestión de si existía una estructura de madera similar a la que existe en Flag Fen (Pryor 1992), o en sitios de Europa Central, como La Tène, durante la Edad del Hierro, desde la cual se arrojarían las armas y ofrendas (Bradley 1990: 97-154). La mayor diferencia entre el Bronce Final y la Edad del Hierro en las zonas del norte de la Europa Atlántica es la mayor institucionalización durante la Edad del Hierro de estos depósitos, que ven aparecer sacrificios humanos intencionales, como es el caso del Hombre de Lindow Moss, el de Tollund o los aparecidos en el interior de los castros u oppida, como Danebury (Bradley 1990), los llamados "bog bodies" (Bahn 1998). En la Edad del Bronce las ofrendas se contextualizan en ritos de paso realizados en puntos nodales de las vías de comunicación, asociados probablemente a costumbres funerarias, y en la Edad del Hierro, se vinculan a ritos de fertilidad agrícola y humana (Bradley 1990: 171-181; Ruiz-Gálvez 1995 b e.p.-b). 


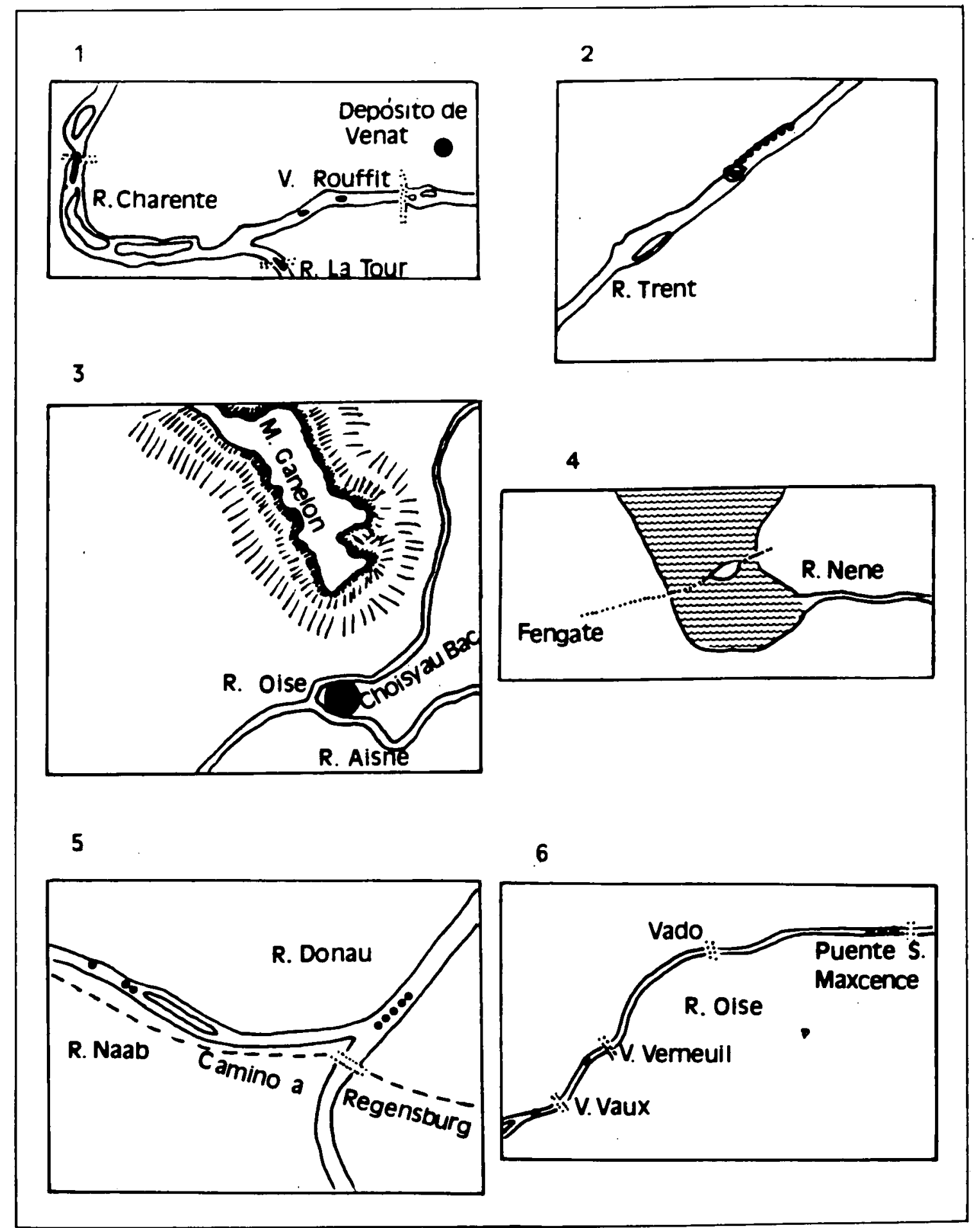

Fig. 2 Hallazgos de metalurgia atlántica europea relacionados con vías de paso y puntos de cruce (Ruiz-Gálvez 1995b): 1.- El depósito de Vénat según Coffyn et al. 1981. 2, 5 y 6.- El sitio de Clifton upon Trent; Regenburg entre el Donau y el Naab; Vados sobre el Oise, según Torbrüge 1970/71. 3.- Choisy au Bac, según Blanchet 1984. 4.- Flag Fen, según Pryor 1991. 


\section{ASUNTOS DE ARISTÓCRATAS}

Estas ofrendas a las aguas durante buena parte del Bronce Final, acompañadas o no de restos humanos en la Península Ibérica, tienen su correspondencia social con la presencia de unas élites guerreras que ostentan el poder en una organización social competitiva, con una ética agonística similar a la de la Iliada y la Odisea (Espejo 1994; Meijer y van Nijf 1992; Finley 1954). La competencia entre los "príncipes" en el intercambio de regalos lleva, en último extremo, a hacer dones a la divinidad, ofrenda máxima ya que no sería devuelta ni rompería el equilibrio del sistema social y económico (Mauss 1963; Finley 1985; Bradley 1990: 1-43).

En la Península, nos encontramos con el mismo sistema social de "príncipes", o "aristoi", que utilizan objetos de prestigio para mantener su estatus en una sociedad jerarquizada (Ruiz-Gálvez e.p.-b) y cada vez más rica, debido sobre todo al aumento y mejora de una ganadería bovina transterminante que está vinculada a zonas húmedas, de pasto, más abundantes tras el aumento de la pluviosidad en la transición del subboreal al subatlántico (Ruiz-Gálvez e.p.-a; Fábregas \& Ruiz-Gálvez 1997; Peña \& Vázquez 1996 ; Méndez 1994).

En la Ría de Huelva y en las representaciones de las estelas decoradas del Suroeste, es más visible la presencia de estas élites. Así lo demuestran las armas (espadas, puñales, carros, escudos con escotadura en V...), y los objetos de adorno, sobre todo fíbulas de codo, pasadores, peines... (Galán 1993). La procedencia o inspiración oriental de la mayoría de estos objetos señala, además de contactos sistematizados en el Bronce Final previos a la colonización (Almagro Gorbea 1986; Ruiz-Gálvez 1995c; Mederos 1996; Díaz Santana e.p.), la asunción por parte de estas élites de nuevos referentes en cuanto al estatus del individuo (como nuevas modas en el vestir), que se reflejan en un nuevo código visual (Cáceres e.p.) que, en el Bronce Final III, utiliza objetos de procedencia atlántica y mediterránea para expresar un estatus social de prestigio igual que en otras áreas de la Europa Atlántica (Ruiz-Gálvez 1995b; Galán 1993).

En Portugal no existen depósitos en las aguas. Sin embargo el depósito del castro de Nossa Senhora da Guia de Baiôes, del s.IX a.C. (Silva 1986; Almagro 1993b,1995b; Ruiz-Gálvez,1995b; Mederos\&Harrison 1996), sí contiene elementos mediterráneos que, aunque descontextualizados como objetos de prestigio, señalan la existencia de estas élites. La presencia de poblados indígenas controlando las vías de comunicación al interior (sobre todo los ríos, - Oliveira 1968; Mayet \& Tavares 1993), indica también un control territorial desde el Bronce Final. La presencia de estos asentamientos estables, más temprana en el norte de Portugal que en la Meseta y en Galicia (Ruiz-Gálvez e.p.-a; Fábregas y Ruiz-Gálvez 1997), explicaría la ausencia de depósitos en las aguas de la costa, aunque sí existen hacia la Meseta, precisamente en los vados de los ríos (gráfico 2). Encontramos en Portugal depósitos de chatarra, muchos de ellos situados en puntos de cruce (Ruiz-Gálvez 1995c), como sucedía en el resto de la Europa Atlántica con los depósitos no votivos, concentrados en las zonas que no poseen las materias primas para elaborar el bronce (Bradley 1990).

En el Valle Medio del Duero, los supuestos enterramientos documentados en silos evidencian una diferenciación social, que corrobora la presencia de objetos de prestigio (armas, torques, fíbulas de codo), tanto por su procedencia foránea (como el hierro, desde el s. IX A.C.) como por la técnica con la que han sido hechos (Delibes et al. 1995: 49-59).

En Galicia predominan las espadas en los ríos, como las del Ulla (Ruiz-Gálvez 1982), las de los Ríos Hío y Sil (Ruiz-Gálvez 1995b), además del tesoro de Caldas de Reyes (Ruiz-Gálvez 1978). Son depósitos de carácter sagrado y profano, enmarcados en la tradición atlántica del Bronce Final de hacer ofrendas a las aguas (Bradley 1990, 1991; Ruiz-Gálvez 1995b). Estas armas y objetos de adorno (torques, arracadas), así como los objetos de prestigio del castro de Torroso, el más antiguo documentado (s. VII A.C.) son el referente de unas élites (Peña 1992, 1995, 1996; Almagro 1993, 1993a) o de una sociedad de tipo heroico (Méndez 1994) que serán más visibles con la monumentalización de los asentamientos, los castros, en el Primer 
Hierro (Parcero 1995; Criado 1993). La ausencia de restos humanos conservados no impide considerar estos depósitos como una desviación de los ajuares desde los túmulos hacia las aguas, de la misma manera que para el resto de la fachada atlántica europea (Fábregas \& Bradley 1995).

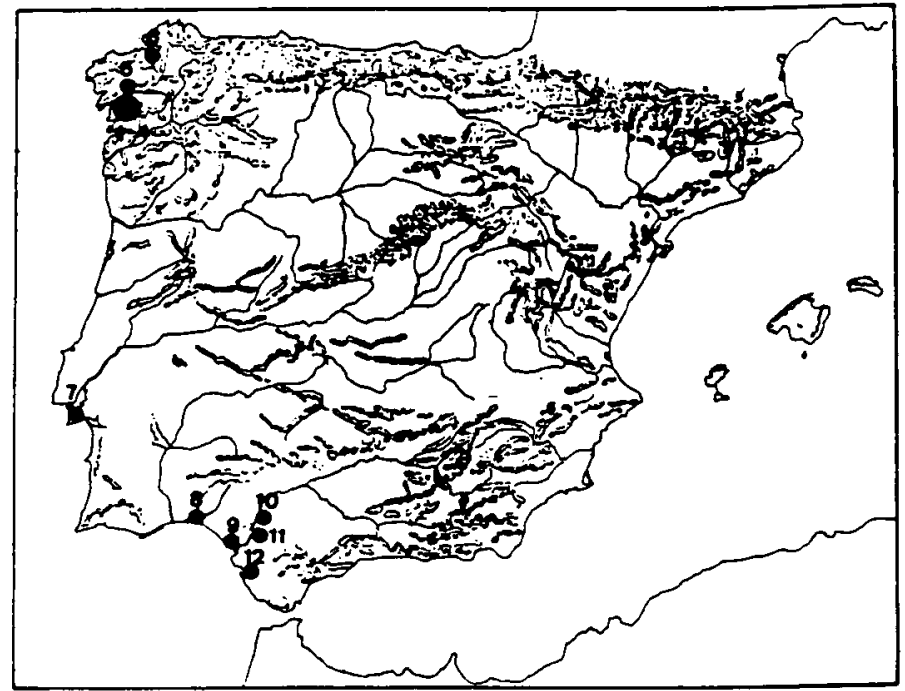

Fig 3: Hallazgos de espadas en las desembocaduras de los ríos peninsulares (Ruiz-Gálvez 1995b): 1-5: río Ulla. 6: río Mero. 7: río Tajo. 8: ría de Huelva. 9-11: río Guadalquivir.

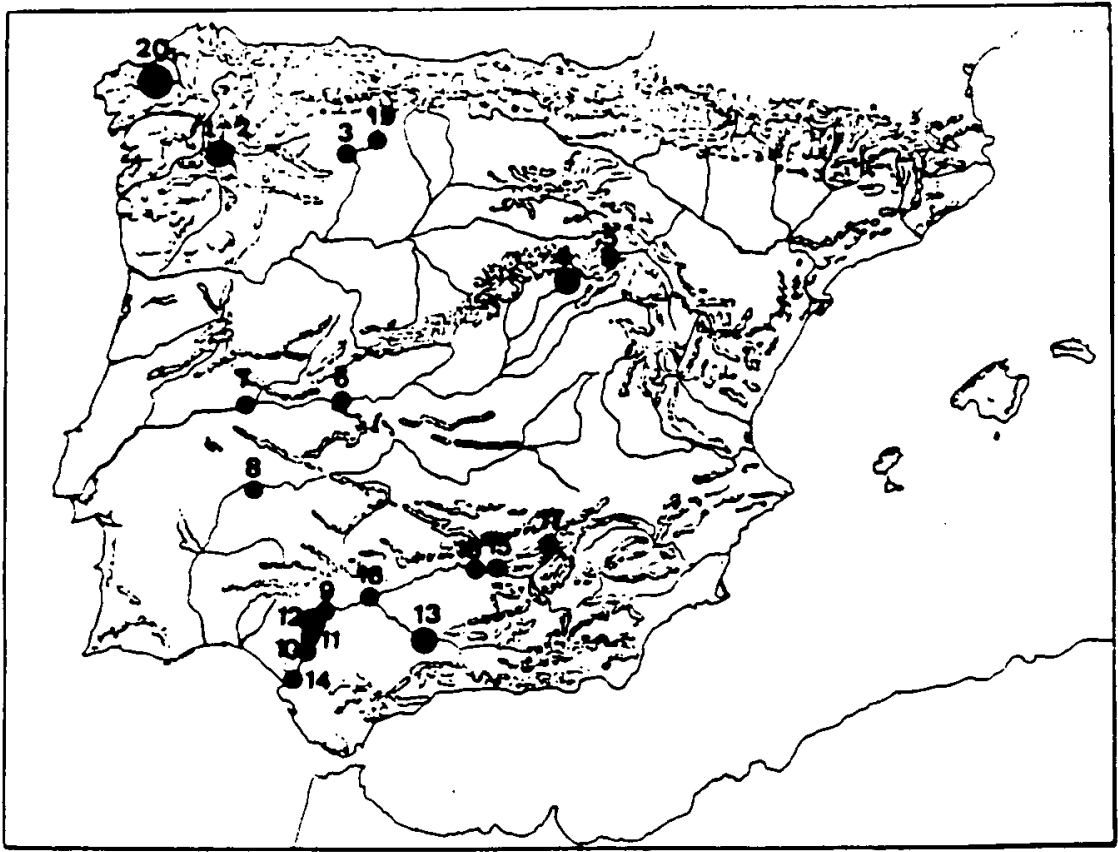

Fig 4: Hallazgos de armas en los vados de los ríos españoles (Ruiz-Gálvez 1995b): 1-2: río Sil. 3: río Úrbigo. 4: río Henares. 5: río Alhama. 6-7: río Tajo. 8: río Guadiana. 9-12 y 15-18: río Guadalquivir. 13: río Geníl. 14: río Guadalete 


\section{PASTOS Y BRAÑAS: UNA VIDA VINCULADA A LOS HUMEDALES}

\subsection{Cabañas y pastizales del Bronce Final}

En el Bronce Final empieza en el Occidente Peninsular la sedentarización de los asentamientos. El surgimiento de una cabaña ganadera de mayor tamaño y proporcionalmente más importante, que socialmente se refleja en la aparición de una aristocracia, indica una economía con predominio de la ganadería de tipo transterminante. La movilidad de los grupos es más reducida que la trashumancia pero necesitaría por igual una serie de pactos intertribales para asegurar el libre tránsito (Ruiz-Gálvez e.p.-a; Fábregas \& Ruiz-Gálvez 1997). Los tesoros, relacionados con puntos de cruce, que M. Ruiz-Gálvez (1995b: 23; e.p.-b) considera como ajuares funerarios, o dotes de mujeres intercambiadas (1992) muestran el control del paisaje por parte de estas élites. Si en las zonas costeras y donde los ríos son las principales arterias de comunicación, los ajuares serían desviados del registro funerario de los enterramientos y túmulos a los puntos nodales de estas vías (los vados y las entradas de los ríos), en el interior pasan a los puertos y pasos de montaña, donde los sucesores del aristócrata guerrero demostrarían la continuidad en la posesión de un paisaje cada vez más territorializado (Bradley 1990; Ruiz-Gálvez 1995b, 1995c e.p.-b; Galán 1993).

En el Suroeste, el dominio del paisaje en esta fase de tránsito hacia la sedentarización se ejerce a través de las estelas decoradas, que sirven de demarcadores territoriales y tribales en los puntos de paso, relacionadas con un entramado de vías de comunicación necesarias para la transterminancia de los grupos (vados de ríos, puertos de montaña) (Galán 1993).

En el Valle del Duero, yacimientos del Bronce Final, tipo Cogotas I, como Cogeces del Monte y Carricastro, en Tordesillas, están situados en zonas de fácil comunicación y con un dominio visual elevado, mostrando una posible jerarquización territorial (Delibes et al. 1995: 53). Durante el Primer Hierro, en Soto de Medinilla, esta jerarquización territorial se plasmará en nuevos yacimientos, con materiales menos perecederos, pero igualmente controlando los puntos nodales de comunicación (Delibes et al. 1995; Romero y Jimeno 1993). Esto explicaría la relativa escasez de depósitos y tesoros o hallazgos aislados en la Submeseta Norte.

En Galicia, en las desembocaduras de los ríos, como en la del Ulla, y en los ríos Hío y Sil, o el tesoro de Caldas de Reyes, se han encontrado armas, sobre todo espadas, y adornos (Ruiz-Gálvez 1978, 1982). Estas deposiciones son similares a las del resto de la Europa Atlántica. Durante el Bronce Final, Galicia y todo el Atlántico ven una reactivación de los contactos que ya habían tenido lugar durante el Neolítico (Ruiz-Gálvez 1990, 1993; Peña 1992, 1995, 1996; Almagro-Gorbea 1986, 1993; Peña \& Vázquez 1996; Bello 1993: 29). El Lusitano se enmarcaría en este proceso como consecuencia de la adopción de una lengua franca por parte de las comunidades del occidente europeo, necesaria para mantener esas rutas comerciales (Ruiz-Gálvez 1990, 1993).

Elementos peninsulares aparecen en zonas de Irlanda, como es el caso de las lúnulas de origen portugués (Waddel 1995; Almagro 1995), y en la Península Ibérica aparecen asadores articulados cuya presencia indica un mismo código de consumo para la fachada atlántica europea, en amortizaciones de riqueza en comidas y festejos (Finley 1985; Galán 1993). El asador articulado de procedencia atlántica de la tumba de Amathus (Chipre), contextualizado también como un objeto de prestigio (Karagheorgis \& lo Schiavo 1989), extiende el alcance de estas rutas hacia el Mediterráneo central (Sherrat \& Sherrat 1991) y muestra la multidireccionalidad de las corrientes de intercambio y el papel activo de las comunidades indígenas del Occidente Peninsular (Mederos 1996; Díaz Santana e.p.).

Durante el Bronce Final II-III, los depósitos de armas y objetos de prestigio (sobre todo vinculados a las aguas), los tesoros de las Submesetas Norte y Sur, y las estelas decoradas o armas y objetos de lujo en tierra firme se sitúan en aquellas zonas que son puntos nodales de las vías de comunicación. Estos puntos 
de paso tienen una doble simbología sagrada y profana, como puntos de tránsito hacia el más allá, lo que explicaría la deposición en ellos de objetos vinculados a ajuares, o a dotes de mujeres intercambiadas entre grupos, como demarcadores territoriales en un paisaje cada vez más conflictivo debido a la transterminancia y a la presión de grupos tribales más competitivos y socialmente más complejos (Bradley 1990; Ruiz-Gálvez 1990, 1995b, e.p.-a, e.p.-b ; Galán 1993).

\subsection{Los castros: monumentos en los valles}

En el Primer Hierro aparecen los primeros asentamientos estables, ya no de materiales perecederos del período anterior. En el Suroeste, la dinámica creada por los asentamientos coloniales no impide que los asentamientos indígenas de la campiña gaditana controlen los accesos al interior, a la vega, que mantendría una cabaña ganadera más especializada (como lo demuestra el mayor tamaño de los animales, posiblemente gracias a mejoras técnicas de origen mediterráneo) demostrando cierta continuidad con el Bronce Final III (Ruiz Mata\&González Rodríguez 1994; Aubet 1994, 1995; Ruiz-Gálvez e.p.-a).

En Portugal la sedentarización es más temprana que en el Noroeste peninsular. Los asentamientos indígenas fortificados se sitúan en las desembocaduras de los ríos controlando la producción y el tránsito de mercancías desde y hacia el interior ya durante el s. VIII a.C. (Oliveira 1968; Mayet y Tavares 1993). Es el caso de Alcácer do Sal y Setúbal, en el Baixo Sado (Mayet y Tavares 1993,), de la Alcáçova de Santarém en la desembocadura del Tajo (de Barros, Cardoso y Sabrosa 1993) y de Conímbriga en el Mondego (Pereira 1993).

En el Valle Medio del Duero, el grupo Soto de Medinilla se desarrolla desde el 1000 A.C.. Se caracteriza por la aparición de nuevos asentamientos, de carácter estable frente a las aldeas de cabañas de Cogotas I. Sin embargo existe cierta continuidad en algunos asentamientos y en el control territorial ya ejercido desde el Bronce Final I-II (vide supra) (Delibes et al. 1995: 49-59).

El Noroeste Peninsular está enmarcado en el Bronce Final dentro de corrientes comerciales o de intercambio que enlazan el Atlántico y el Mediterráneo, ya sea hacia el Sur y las costas portuguesas (Peña 1995, 1996), o hacia el Norte, a Europa Central a través de Francia y Gran Bretaña (Ruiz-Gálvez 1990, 1993), que parecen reducir su importancia y alcance en la etapa formativa de la Cultura Castreña (Peña\&Vázquez 1996). El asentamiento más temprano es el castro de Torroso, del s. VII A.C. (Peña 1992). La presencia de objetos de prestigio, como la placa de cinturón damasquinada, o el uso del hierro (aunque sea en útiles) muestra cierta complejidad social y un gran dinamismo de la comunidad (Peña Santos 1992) en las corrientes de intercambio (Peña y Vázquez 1996; Peña 1995, 1996). Según Peña Santos (1992) la presencia de cabañas en su interior , más que responder a una estratigrafía demuestra la contemporaneidad de éstas con las viviendas de mampostería. Esto no descarta una mayor sedentarización para las comunidades del Bronce Final del Noroeste. Tanto Torroso como los yacimientos del Valle del Ulla (Carballo 1995), de Ferrol (Parcero 1995) y del Río Pedriña en la Sierra de O Bocelo (Méndez 1994) están orientados a las tierras de cultivo de estos valles en su fase inicial. Orientan su visibilidad y los accesos hacia las brañas, que darían los pastos para una cabaña ganadera estabulada frente a la transterminante del periodo anterior (Méndez 1994; Carballo 1990; Peña 1992), y para una agricultura de rozas más avanzada que la del Bronce (Méndez 1995 vs. Peña y Vázquez 1996). Las zonas húmedas de los valles constituyen la base económica de estas comunidades, y hacia su control se orienta la dispersión de los castros en su fase inicial (Méndez 1995). Existe continuidad respecto al período anterior, ya que las armas en las aguas se encuentran en puntos determinados de estos ríos, y los últimos túmulos y los petroglifos ejercen el mismo papel de demarcadores territoriales (Méndez 1995; Carballo 1990) que se supone para las estelas del Suroeste (Galán 1993). Las diferencias regionales, como la mayor concentración de petroglifos en la zona meridional de Galicia, señalan diferencias en los sistemas de control territorial por parte de los grupos y de las élites (Carballo 1990). 
Durante el Bronce Final el control territorial se realizaría a través de rituales de deposición de armas y objetos de prestigio, vinculados quizá al mundo funerario (Bradley 1990; Ruiz-Gálvez 1995b) y a través de los petroglifos y los últimos túmulos como referencia visible en el paisaje (Fábregas\&Bradley 1995; Carballo 1990; Bello 1995; Bradley, Criado y Fábregas 1994; Méndez 1994). La aparición de los castros supone la "monumentalización" de la presencia del grupo en el territorio y se convierte en el referente visual por excelencia (Parcero 1995; Criado 1993).

El papel de los ríos en Galicia sigue siendo el de vías de comunicación y de dispersión de los asentamientos. En el Bronce Final, las aldeas de cabañas se situarían más cerca de las zonas húmedas, en las brañas más resguardadas (Méndez 1990). La "revolución de los productos secundarios" da mayor importancia a la ganadería vacuna, y a los humedales como base económica (Méndez 1994; Peña 1992, 1996; Carballo 1990; Ruiz-Gálvez e.p.-a). Durante el Primer Hierro, cobran mayor importancia las tierras de labradío y los castros se situarán en los mismos valles, pero cerca de estas tierras, con un menor carácter defensivo y sin alejarse de las zonas de pasto (Méndez 1994).

\section{CONCLUSIONES}

La transición del subboreal al subatlántico durante el Bronce Final provoca en la fachada atlántica europea un aumento de la pluviosidad y de las condiciones de humedad. Las comunidades indígenas mantienen una economía de carácter transterminante. La cabaña ganadera muestra gran importancia del ganado bovino a juzgar por la importancia proporcional de sus restos y el mayor tamaño de los animales, lo que indica una especialización ganadera. La transterminancia requiere una movilidad menor que la trashumancia típica de la Edad del Bronce, pero necesita unas redes de contactos que aseguren la movilidad de los grupos.

Los asentamientos del Bronce Final en el Occidente Peninsular son aldeas de cabañas, que muestran en Soto de Medinilla una importante estratigrafía horizontal, señalando la reutilización de los asentamientos. En el Suroeste, las comunidades indígenas de la campiña gaditana controlan a la llegada de los colonos fenicios la campiña y vega gaditanas. En Portugal tienen mayor importancia los ríos como arterias de comunicación, y la sedentarización, desde el s. VIII a.C., se produce más temprano que en el Noroeste peninsular. Aquí la transterminancia se convierte en el medio óptimo de aprovechamiento del medio y la aparición de los castros se documenta en Torroso, en el s. VII A.C., conviviendo en sus momentos iniciales las cabañas de materiales perecederos con las viviendas de mampostería.

La existencia de una cabaña ganadera de mayor importancia provoca la aparición del aristos, perteneciente a una élite guerrera que controla la producción, la redistribución y que representa a la comunidad. Dentro de una concepción sustantivista de los sistemas económicos, las élites se relacionan a través del intercambio de dones, creando vínculos de sangre y proporcionando el marco de relaciones intertribales necesario para la movilidad estacional del ganado.

La ausencia de evidencias funerarias desde mediados del II milenio a.C. en la fachada atlántica europea lleva a muchos autores a considerar que los abundantes depósitos de armas, adornos y objetos de prestigio en general se corresponden a una desviación del ajuar del difunto desde los túmulos y enterramientos anteriores hacia las aguas. Restos humanos parciales serían arrojados a las aguas de los ríos. El ritual de paso tendría lugar en lugares estratégicos por servir de puntos de paso obligado, tal como las desembocaduras del río Ulla, o la Ría de Huelva, o en los vados. Los tesoros o depósitos en tierra firme, se consideran también ajuares, o dotes de mujeres que se intercambian entre las élites, en un sistema de dones o regalos de carácter recíproco, depositados en lugares estratégicos de las vías de comunicación sobre los que el grupo o la élite local desea marcar un control. 
En el Suroeste Peninsular, las estelas decoradas suponen un lenguaje visual de control territorial, en el que las diferencias regionales en la iconografía de las estelas representarían diferencias grupales. Las estelas diademadas serían el correspondiente a las dotes de las mujeres intercambiadas.

En el Noroeste Peninsular, los petroglifos, que se concentran en el área meridional, son otra forma de control territorial por parte de las élites que conforman una sociedad jerarquizada, de tipo heroico. Los últimos túmulos son otro tipo de referente visual del dominio de un grupo sobre el territorio, generalmente los valles de los ríos.

La aparición durante la Primera Edad del Hierro de los primeros asentamientos estables en el Occidente Peninsular se corresponde en el registro arqueológico con la paulatina desaparición de los depósitos vinculados a las aguas. Las Culturas de los Castros de la fachada atlántica se vinculan generalmente a un sustrato cultural pre- o protocelta, en el que las aguas juegan un papel esencial como zonas onfálicas en la cosmogonía de las etnias. La continuidad respecto a estas deposiciones del Bronce Final suscita el debate de la existencia de este sustrato protocelta a partir de orígenes indoeuropeos y de su llegada, ya sea a través de las redes atlánticas o a través de los Pirineos y el interior peninsular.

El castro es el referente visible por excelencia de la presencia del grupo humano y de su posesión del territorio. En el Valle Medio del Duero, el Grupo Soto de Medinilla comienza desde el 1000 A.C. Una cabaña ganadera bovina lo asocia a terrenos de pastos para el ganado. Los asentamientos se sitúan en lugares estratégicos en cuanto a visibilidad y defensa, pero como en el Bronce Final cerca de zonas húmedas.

La vega y campiñas gaditanas son controladas desde el Bronce Final por aldeas indígenas, que continuarán ejerciendo ese dominio durante la etapa colonial. Una base económica agrícola y ganadera mantiene a unas élites que desvían la fuerza de producción hacia la creación de excedentes de materias primas con las que mantener los contactos con los colonos.

Los castros portugueses continúan la dinámica de dominio territorial y de las vías de comunicación que había comenzado ya en el Bronce Final. En Galicia, por el contrario, los castros aparecen de forma más tardía, y vinculados de manera más clara a las zonas húmedas de los valles de los ríos, las brañas. Su base económica, que se creía esencialmente ganadera, hoy es considerada mixta, complementada con una agricultura de rozas más desarrollada que durante el Bronce Final I-II. Los castros se sitúan en lugares de fácil defensa, sobre todo en la fase inicial, pero sus defensas arquitectónicas se vinculan a la idea de monumentalidad, de referente visual del dominio total del grupo humano sobre el territorio. Los valles de los ríos y sus brañas parecen determinar en última instancia la ubicación y la orientación de los asentamientos. 


\section{BIBLIOGRAFÍA}

ALDHOUSE-GREEN, M. (1993): "La religión Celta" , en M. ALMAGRO-GORBEA y G. RUIZ ZAPATERO, (eds.), Los Celtas: Hipania y Europa, Madrid, Edit. Actas, pp 451- 472.

ALMAGRO-GORBEA, M. (1995): "Ireland and Spain in the Bronze Age", en J. WADDELL y E. SHEE TWOHIG (eds.), Ireland in the Bronze Age, Proceedings of the Dublin Conference, April, 1995. Dublin, Stationery Office, pp.136-148.

ALMAGRO-GORBEA, M. (1993a): "Los Celtas en la Península Ibérica: origen y personalidad cultural”, en M. ALMAGRO-GORBEA y G. RUIZZAPATERO, (eds.), Los Celtas: Hispania y Europa , Madrid, Edit. Actas, pp. 121-172.

ALMAGRO-GORBEA, M. (1986): "Bronce Final y Edad del Hierro", en Historia de España, Prehistoria, Madrid, Gredos, pp.341-532.

ALMAGRO-GORBEA, M. (1993): "Secuencia Cultural y Etnogénesis del centro y noroeste de la Península Ibérica”, en Actas del XXII Congreso Nacional de Arqueología, Vigo, 1993 (I), pp. 121-138.

AUBET, M.E. (1994): Tiro y las colonias fenicias de Occidente, Edit. Crítica, Sabadell.

AUBET, M. E. (1995): "El comercio fenicio en Occidente. Balance y perspectivas", I Fenici: ieri oggi domani. Ricerche, scoperte, progetti (Roma 3-5 Marzo 1994), Roma, Academia Nazionale dei Lincei, Consigli Nazionale delle Ricerche, pp. 227-243.

BAHN, P. (1997): "Bodies of the bogs", Archaeology, July/ August, n 4, pp. 62-67.

BARROS (de), L.; CARDOSO, J.L.; SABROSA, A., (1993): "Os fenicios na margem sul do Tejo. Economía e integraçao cultural no povoado do Almaraz-Almada”, en A.A. TAVARES DE SILVA, editor, $O s$ fenicios no territorio portugués, Estudos Orientais, IV, Instituto Oriental, Universidad Nueva de Lisboa, pp.143-182.

BELLO, J.M. (1993): “Autoctonismo vs. relaciones en el Megalitismo Noroccidental. El caso de los monumentos de Dombate”, Actas del XXII Congreso Nacional de Arqueología, I, Vigo, 1993, pp. 25-32.

BRADLEY, R.; CRIADO, F.; FÁBREGAS, R. (1994): "Los petroglifos como forma de apropiación del espacio: algunos ejemplos gallegos", Trabajos de Prehistoria, 51 (II), pp. 159-168.

BRADLEY, R. \& GORDON, K. (1988): "Human skulls from the river Thames, their date and significance", Antiquity, 62, pp. 503-509.

BRADLEY, R. (1990): The Passage of Arms: an archaeological analysis of prehistoric hoards and votive deposits, Cambridge University Press.

CÁCERES, Y. (e.p.): "Cerámicas y tejidos: sobre el significado de la decoración geométrica del Bronce Final en la Península Ibérica".

CRIADO, F. (1993): "Límites y posibilidades de la Arqueología del Paisaje”, SPAL, 2, pp. 9-55.

DELIBES, G.; ROMERO, A.; MORALES, A. (eds.) (1995): Arqueología y medio ambiente. El primer milenio antes de Cristo en el Duero Medio, Junta de Castilla y León, Consejería de Cultura y Turismo.

DÍAZ SANTANA, B. (e.p.): "Contactos precoloniales durante el Bronce Final en el Occidente Peninsular: el papel activo de las comunidades indígenas".

ESPEJO, C., (1994): "Religión e ideología en Homero", Studia Historica-Historia Antigua, vol. XIII, pp. 9-20.

FÁBREGAS, R. \& RUIZ-GÁLVEZ, M. (1997): "El Noroeste de la Península Ibérica en el III-II milenios: propuestas para una síntesis”, Saguntum, (PLA V), 30, pp. 191-216.

FÁBREGAS, S.; BRADLEY, R., (1995): "El silencio de las fuentes: prácticas funerarias en la Edad del Bronce del Noroeste y su contexto europeo”, Complutum, 6, Madrid , pp. 153-166.

FÁBREGAS, R. (1995): "El fenómeno tumular en el Bronce del Noroeste", en Actas del XXII Congreso Nacional de Arqueología , Vigo, 1993 (I), pp. 85-92. 
FINLEY, M.I. (1954): The world of Odysseus, traducción al castellano, El Mundo de Odiseo, F.C.E., México, 1995.

FINLEY, M.I. (1985): The Ancient Economy, London, The Hugarth Press.

GALÁN, E. (1993): Estelas, paisaje y territorio en el Bronce Final del Suroeste de la Península Ibérica, Editorial Complutense, Madrid.

HOMERO: Odisea, Barcelona, Editorial Planeta, 1980.

MARCO, F. (1993): "La religiosidad en la Céltica hispana", en M. ALMAGRO-GORBEA y G. RUIZ ZAPATERO, editores, Los Celtas: Hispania y Europa, Madrid, Edit. Actas, pp. 477-512.

MAUSS, M., (1963): "Essai sur la don. Forme et raison de l'echange dans les societés archaïques. L'année sociologique", en M. MAUSS, ed., Sociologie et anthropologie, París, P.U.F., pp.145-171.

MAYET, F.;TAVARES, A.A. (1993): "Presencia fenicia no Baixo Sado", en A.A. TAVARES, (ed.), Os fenicos no territorio portugués, Estudos Orientáis, IV, Instituto Oriental, Universidad Nueva de Lisboa, pp. $127,142$.

MEDEROS, A. (1996): "La conexión levantino chipriota. Indicios de comercio atlántico con el Mediterráneo Oriental durante el Bronce Final (1150-950 a.C.), Trabajos de Prehistoria, 53, $\mathrm{n}^{\circ}$ 2, pp. 5-115.

MEIJER, F. \& van NIJF, G. (1992): Trade transport and the society in the Ancient World, A Sourcebook, London, Routledge.

MÉNDEZ, F. (1994): "La domesticación del paisaje durante la Edad del Bronce gallego", Trabajos de Prehistoria, 51, 1994, pp.77-94.

OLIVEIRA, A.H. de(1968): Introduçao à Història da Agricultura em Portugal, Lisboa, Ed.Cosmos.

PARCERO, C. (1995): "Elementos para el estudio de los paisajes castreños del noroeste peninsular", Trabajos de Prehistoria , 52 (I) , pp. 127-144.

PEÑA, A. (1992): Castro de Torroso (Mos, Pontevedra). Síntesis de las memorias de las campañas de excavaciones 1984-1990, Xunta de Galicia, Arqueoloxía/Memorias,11.

PEÑA, A. (1992a): "El primer milenio a.C. en el área gallega: génesis y desarrollo del mundo castreño a la luz de la arqueología", en M. ALMAGRO-GORBEA y G. RUIZZAPATERO (eds.), Paleoetnología de la Península Ibérica ,Complutum, 2-3, Madrid, pp.373-394.

PEÑA, A. (1996): "Aspectos de la génesis y evolución de la Cultura Castrexa de Galicia”, Complutum, Extrta $6(1)$, pp. 255-262.

PEÑA, A. (1995): "La secuencia cultural del mundo castrexo galaico", en J.M. HIDALGO CUÑARRO (Coord.), A Cultura Castrexa galega a debate, (Actas del Curso de Verano de la Universidad de Vigo, Tui, 1995), Instituto de Estudios Tudenses, Vigo, pp. 65-103.

PEÑA, A. de la\& VÁZQUEZ VARELA, J.M. (1996): Aspectos de la génesis y evolución de la Cultura Castrexa de Galicia, Complutum, Extra 6 (I), pp. 255-262.

PEREIRA, I., (1993): “Figueira da Foz. Santa Olaia.", en A.A. TAVARES, ed., Os fenicios no territorio portugués, Estudos Orientais, IV, Instituto Oriental, Universidad Nueva de Lisboa, pp. 285-301.

PRYOR, F. (1992): "Discussion: the Fengate / Northey landscape", Antiquity, 66, pp. 518-531.

ROMERO, F. y JIMENO, A. (1993): "El valle del Duero en la antesala de la Historia. Los grupos del Bronce Medio-Final y Primer Hierro”, en M. ALMAGRO-GORBEA y G. RUIZZAPATERO, (eds.), Los Celtas: Hispania y Europa, Madrid, Edit. Actas, pp.175-219.

RUIZ-GÁLVEZ,M. (1995a): "La Ría en relación con la metalurgia de otras regiones pè̀ninsulares durante el Bronce Final", en RUIZ-GÁLVEZ, M. (ed.), Ritos de paso y puntos de paso: la Ría de Huelva en el mundo del Bronce Final Europeo, pp. 59-68.

RUIZ-GÁLVEZ, M. (1995b): "Depósitos del Bronce Final: ¿Sagrado o profano? ¿Sagrado y, a la vez, profano?", en M. RUIZ-GÁLVEZ, (ed.), Ritos de paso y puntos de paso: la Ría de Huelva en el mundo del Bronce Final europeo, Madrid, Editorial Complutense, Complutum Extra 5, pp.21-32. 
RUIZ-GÁLVEZ, M. (1995c): "El significado de la Ría de Huelva en el contexto de las relaciones de intercambio y de las transformaciones producidas en la transición Bronce Final / Edad del Hierro", en M. RUIZ-GÁLVEZ, (ed.), Ritos de paso y puntos de paso: la Ría de Huelva en el mundo del Bronce Final europeo, Madrid, Editorial Complutense, Complutum Extra 5, pp. 129-155.

RUIZ-GÁLVEZ, M. (1995d): "Cronología de la Ría de Huelva en el marco del Bronce Final de Europa Occidental”, en RUIZ-GÁLVEZ, M. (ed.), Ritos de paso y puntos de paso: la Ría de Huelva en el mundo del Bronce Final europeo, pp.79-84.

RUIZ- GÁLVEZ, M. (1990): “Canciones del muchacho viajero”, Veleia, 7, pp.79-103.

RUIZ-GÁLVEZ, M. (1992): "La novia vendida: orfebrería, herencia y agricultura en la Protohistoria de la Península Ibérica”, SPAL, 1, pp.219-251.

RUIZ-GÁLVEZ, M. (1993): "El Noroeste de la Península Ibérica en el contexto de la Prehistoria Reciente de Europa Occidental", Actas del XXII Congreso Nacional de Arqueología, I, Vigo, pp. 11-18.

RUIZ-GÁLVEZ, M. (e.p.-a): "Settlement patern and socioeconomic changes in the Bronze Age / Iron Age transition of the Spanish Meseta and Southwest", en Mench und umwelt im bronze-zeit europas, Berlín, 1992.

RUIZ-GÁLVEZ, M. (e.p.-b): “Lieux de passage, no man's land, et lieux neutres d'echange”, en L'atelier du bronzier, Dijon, Febrero, 1998.

RUIZ-GÁLVEZ, M. (1978): “El tesoro de Caldas de Reyes”, Trabajos de Prehistoria, 35, pp. 173-192.

RUIZ-GÁLVEZ, M. (1982): “Nueva espada dragada en el río Ulla. Armas arrojadas a las aguas”, ElMuseo de Pontevedra, $\mathrm{n}^{\circ}$. XXXVI, En memoria de Alfredo García Alen, pp. 181-196.

RUIZ MATA , D. (1993): "Los fenicios de la época arcaica -siglos VIII/VII a.C.- en la Bahía de Cádiz. Estado de la cuestión”, en A.A. TAVARES, ed., Os fenicios no territorio portugués, Estudos Orientais, IV, Instituto Oriental, Universidad Nueva de Lisboa, pp.23-72.

RUIZ MATA, D.; GONZÁLEZ RODRÍGUEZ, R. (1994): “Consideraciones sobre asentamientos rurales y cerámicas orientalizantes en la campiña gaditana", $S P A L, 3$, Madrid, pp. 209-256.

SILVA, C.F.A. (1986): A cultura castrexa do Noroeste de Portugal, Museo Arqueológico da Citania de Sanfins, Paços da Ferreira.

SHERRAT, A. \& S. (1991): "From luxuries to commodities: the nature of Mediterranean Bronze Age trading siystems", en N. H. GALE, editor, Bronze Age trade in the Mediterranean, Studies in Mediterranean Archaeology XC, Sweden, pp.351-386.

WADDELL, J. (1995): "Celts, celtisation, and the Irish Bronze Age”, en J. WADDELL y E. SHEE (eds.), Ireland in the Bronze Age, Procceedings of the Dublin Conference, April, 1995, Dublin, Stationery Office, pp. 158-169. 